\title{
Progranulin as a Potential Therapeutic Target in Immune-Mediated Diseases
}

\author{
Yue-Jiao Lan ${ }^{1,2}$ \\ Napoleon Bellua Sam ${ }^{3}$ \\ Ming-Han Cheng' \\ Hai-Feng Pan ${ }^{4,5}$ \\ Jian Gao' \\ 'Pediatric Translational Medicine \\ Institute, Shanghai Children's Medical \\ Center, School of Medicine, Shanghai Jiao \\ Tong University, Shanghai, People's \\ Republic of China; ${ }^{2}$ The Second Affiliated \\ Hospital, Dalian Medical University, \\ Dalian, Liaoning, People's Republic of \\ China; ${ }^{3}$ Department of Medical Research \\ and Innovation, School of Medicine, \\ University for Development Studies, \\ Tamale, Ghana; ${ }^{4}$ Department of \\ Epidemiology \& Biostatistics, School of \\ Public Health, Anhui Medical University, \\ Hefei, Anhui, People's Republic of China; \\ ${ }^{5}$ Inflammation and Immune Mediated \\ Diseases Laboratory of Anhui Province, \\ Hefei, People's Republic of China
}

Correspondence: Jian Gao Pediatric Translational Medicine Institute, Shanghai Children's Medical Center, School of Medicine, Shanghai Jiao Tong University, Shanghai, 200120, People's

Republic of China

Email gaojian@scmc.com.cn; gaojianayfy@163.com

Hai-Feng Pan

Department of Epidemiology and

Biostatistics, School of Public Health,

Anhui Medical University, 8I Meishan

Road, Hefei, Anhui, 230032, People's

Republic of China

Email panhaifeng@ahmu.edu.cn

\begin{abstract}
Progranulin (PGRN), a secretory glycoprotein consisting of 593 amino acid residues, is a key actor and regulator of multiple system functions such as innate immune response and inflammation, as well as tissue regeneration. Recently, there is emerging evidence that PGRN is protective in the development of a variety of immune-mediated diseases, including rheumatoid arthritis (RA), inflammatory bowel disease (IBD), type 1 diabetes mellitus (T1DM) and multiple sclerosis (MS) by regulating signaling pathways known to be critical for immunology, particularly the tumor necrosis factor alpha/TNF receptor (TNF- $\alpha$ /TNFR) signaling pathway. Whereas, the role of PGRN in psoriasis, systemic lupus erythematosus (SLE) and systemic sclerosis (SSc) is controversial. This review summarizes the immunological functions of PGRN and its role in the pathogenesis of several immune-mediated diseases, in order to provide new ideas for developing therapeutic strategies for these diseases.
\end{abstract}

Keywords: PGRN, TNF- $\alpha$, TNFR, immune-mediated diseases, therapeutic

\section{Introduction}

The immune system has several fundamental functions, namely protecting against invasion by pathogenic micro-organisms, removing mutated somatic cells, and regulating the suitable immune system's reaction intensity to antigenic stimuli. ${ }^{1}$ In relation to physiological and pathological conditions, the immune system is inseparable from the changes in inflammatory mechanisms. When the body is attacked by injury or pathogenic micro-organisms irruption, it is often accompanied by acute inflammation, a normal safeguarding response. However, uncontrolled inflammatory responses and sustained immune responses may result in immune system disorders such as rheumatoid arthritis (RA) and diabetes mellitus as well as inflammatory bowel disease (IBD). ${ }^{2}$ Although the release of cytokines for immunity and inflammation is crucial to the immune functions, hyper-function of cytokines such as tumor necrosis factor alpha (TNF- $\alpha$ ) may damage host tissues, and its mobilization against self-targets causes the development of autoimmune disorders. TNF- $\alpha$ is at the top of the reaction of inflammatory cascade, the TNF- $\alpha$ /TNFR signaling pathway coordinates a large number of inflammatory processes, and is pivotal for the occurrence and development of sundry inflammation-induced immune-mediated diseases. As a secreted molecule with some cytokine-like properties, progranulin (PGRN) is a unique ligand of TNF receptor (TNFR), which plays its anti-inflammatory action mainly through TNF Receptor 1 (TNFR1) and stimulus $\mathrm{CD} 4{ }^{+} \mathrm{CD} 25^{+}$Foxp $^{+}{ }^{+}$regulatory T cells (Treg) activity by binding to TNF Receptor 2 


\section{Graphical Abstract}



(TNFR2) to limit the role of TNF- $\alpha$ in some immunemediated diseases, such as RA and IBD. ${ }^{3-6}$ In addition to the effect on maintaining Treg function, PGRN deficiency also interferes with the immune response of other $\mathrm{T}$ cells such as T-helper 1 (Th1) and T-helper 17 (Th17), PGRN influences Janus kinase/signal transducer and activator of transcription (JAK/STAT) pathway to reduce cell response of Th1 and Th17 and cytokines secretion in vitro and in vivo. ${ }^{7}$ PGRN is cleaved by elastase to yield discrete fragments named Granulin (GRN) in vitro. The GRN acts as an essential role in defense infection in the innate immunity. ${ }^{8}$ The innate immunity belongs to the immune system, has been considered as the first powerful defense against the invasion of various pathogenic microorganisms and foreign bodies. ${ }^{9}$ GRN assists in recruiting of $\mathrm{CpG}$ oligonucleotides (CpG-ODNs) in macrophages by binding to Toll-like receptor 9 (TLR9), reinforcing innate immunity against bacteria invasion. ${ }^{8,10,11}$ For example, PGRN can exacerbate SLE severity by enhancing TLR9 signaling. ${ }^{12}$ Therefore, PGRN may be the primary regulators of inflammation and autoimmunity and may serve as biomarkers as well as indicating a promising and novel therapeutic concept to treat diverse inflammatory immunemediated diseases. In this review, we will briefly summarize the immunological functions of PGRN and its role in immune-mediated diseases, and highlight its potential as a therapeutic target for these diseases.

\section{Immunological Functions of PGRN}

PGRN is similarly known as proepithelin (PEPI), PC-cellderived growth factor (PCDGF), Granulin-epithelin precursor (GEP), and GP88. ${ }^{13}$ It is a protein of 12 coding exons transcribed from the gene Granulin (GRN), located on the human chromosome 17q21.32. ${ }^{13,14}$ The architecture of PGRN is made up of 7.5 cysteine-rich motif (CX5-6CX5CCX8CCX6C CXDX2HCCPX4CX5-6C, X: any amino acid) with a unique bead-like structure. ${ }^{14}$ The molecular structure of each GRN is a domain of six parallel stacks of $\beta$-hairpins linked by six disulfide bridges. ${ }^{15,16}$ The disulfide bridges are crucial to maintain appropriate protein folding and unique confirmation. The $68.5 \mathrm{kDa}$ relative molecular mass PGRN may be lysed by a variety of proteolytic enzymes. Expression of the PGRN may be discovered in rapid proliferating cells, which comprise epithelial cells, ${ }^{14,17}$ immune cells, ${ }^{18}$ macrophages, ${ }^{19}$ neuronal cells, ${ }^{20}$ adipocytes, ${ }^{21}$ fibroblastic cell, ${ }^{14,17}$ and chondrocytes ${ }^{22}$ (Figure 1).

PGRN not only plays a fundamental role in tumorigenesis, ${ }^{23,24}$ but also is a vital immunomodulator. In relation to disease types and key signaling pathways, PGRN functions as a double-edged sword in playing the pro- 


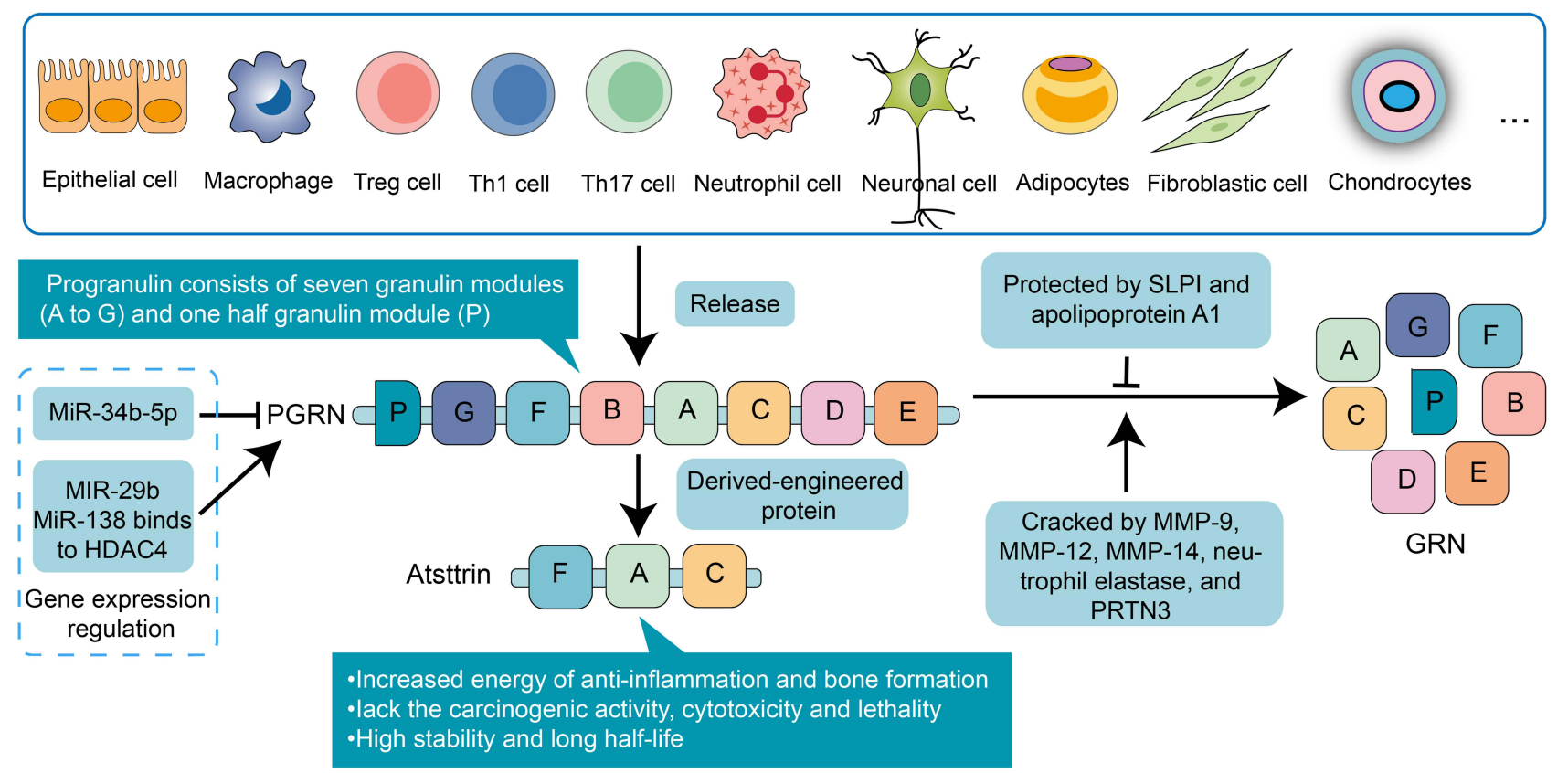

Figure I The structure, source and intervention of PGRN in the autoimmune system. The architecture of PGRN is made up of 7.5 cysteine-rich motif and mainly distributes in various cell lines. Some microRNAs specifically stimulate or inhibit PGRN activation. The SLPI and apolipoprotein AI can bind to it to protect it against proteolytic degradation by MMP-9, MMP-12, MMP-I4, neutrophil elastase, and PRTN3. Atsttrin: an engineered protein consists of the FAC domain of PGRN.

Abbreviations: PGRN, Progranulin; GRN, Granulin; HDAC4, histone deacetylase 4; SLPI, secretory leukocyte protease inhibitor; MMP-9, matrix metalloproteinase-9; PRTN3, proteinase 3.

inflammatory role and anti-inflammatory role in immunemediated diseases. Furthermore, PGRN may be an effective bone morphogenetic protein that participates in the development of related immune diseases involving joints or arthritis complications such as systemic lupus erythematosus (SLE), RA and psoriasis. ${ }^{25}$ Nevertheless, the proper function regulated by PGRN may vary relying on the pathology and physiology context of the disease (Figure 2).

\section{Inflammation}

During inflammation, neutrophils and macrophages release proteases such as matrix metalloproteinase-9 (MMP-9), ${ }^{26}$ MMP-12, ${ }^{27}$ MMP-14,${ }^{28}$ neutrophil elastase, ${ }^{29}$ and proteinase 3 (PRTN3) ${ }^{30}$ in which PGRN may be subjected to degradation to yield individual, approximately 6-kDa GRN fragments. However, GRN often presents the opposite effect to PGRN and neutralizes the complete PGRN's antiinflammatory effects. ${ }^{31}$ Furthermore, GRN stimulates secretion of interleukin-8 (IL-8) by epithelium cells in multiple sclerosis (MS), a primary chemotactic agent for neutrophils and monocytes, while PGRN is short of this activity. ${ }^{31,32}$

\section{Anti-Inflammation}

Conversely, the protection of anti-inflammatory properties of PGRN depends on its combined proteins such as the secretory leukocyte protease inhibitor ${ }^{31}$ and apolipoprotein A1, ${ }^{33,34}$ which bind with full-length PGRN against protease hydrolysis. ${ }^{35}$ Compared with its pro-inflammatory mechanism, its inhibitory effect on inflammation has received greater attention. The central anti-inflammatory role of PGRN is to mediate the TNF- $\alpha$ /TNFR signaling pathway through binding to the slender cysteine-rich domain 2 (CRD2) and CRD3 of TNFR1 and TNFR2 extracellular region. ${ }^{36}$ Moreover, the binding affinity of PGRN and TNFR1 is equivalent to that of TNF- $\alpha$, while the binding affinity of PGRN and TNFR2 is nearly 600 times that of TNF- $\alpha$. Among them, PGRN binds to TNFR1 and activates extracellular regulated protein kinase 1 and 2 (ERK1/2) and phosphatidylinositol 3 kinases/protein kinase B (PI3K/AKT) pathways to competitively interfere with activation of TNF- $\alpha$ mediated nuclear factor of kappa (NF-kB) inflammatory pathway. ${ }^{5,11,36,37}$ Besides, PGRN and its derivative Atsttrin, directly combine to death receptor 3 (DR3), the highest homology to TNFR, to block the binding of DR3 and TNF-like ligand 1A (TL1A) to play a supplementary anti-inflammatory role. ${ }^{38,39}$ Additionally, recent findings suggested that cytokine IL-10 with antiinflammatory properties predominantly from Treg cells, was a crucial mediator in PGRN-mediated antiinflammation. ${ }^{40}$ Again, PGRN expands and activates 




Figure 2 Immunological functions of PGRN. Four main immunological functions of PGRN: Pro-inflammatory: PGRN can be subjected to degradation into GRN fragments by MMP-9, MMP-I2, MMP-14, neutrophil elastase, and PRTN3, GRNs stimulate secretion of IL-8 by epithelium cells in MS; Anti-inflammatory: PGRN binds to TNFRI and activates ERKI/2 and PI3K/AKT pathways to inhibit TNF- $\alpha$ activated NF-KB inflammatory pathway which promotes the release of pro-inflammatory cytokines, mainly IL-6; PGRN selectively inhibits expression and release of CXCL9 and CXCLI0 induced by TNF- $\alpha$ in a TNFRI dependent manner; Combination of PGRN and TNFR2 will ameliorate chronic inflammatory disorders via FOXO4-STAT3-dependent IL-10 production in Tregs; Induce chondrogenesis and cartilage repair: PGRN activates the ERK/ JunB signaling pathways; PGRN may inhibit cartilage loss bone resorption mediated by NF-KB pathway in a TNFR I-dependent manner; Inhibiting ADAMTS-7/ADAMTS- I2mediated COMP degradation and TNF- $\alpha$-induced ADAMTS-7/ADAMTS-12 expression; Regulating of TLR9 signaling pathway.

Abbreviations: FOXO4, forkhead box protein O4; STAT3, signal transducer and activator of transcription 3; Treg, regulatory T cells; Ikk, I kappaB kinase; IrB, irbesartan; PRTN3, proteinase 3; MMP-9, matrix metalloproteinase-9; IL-8, interleukin-8; ERKI/2, extracellular regulated protein kinase I and 2; PI3K, phosphatidylinositol 3 kinases; AKT, protein kinase B; NF- $\mathrm{KB}$, nuclear factor of kappa; Tregs, regulatory T cells; ADAMTS-7, A disintegrin and metalloproteinase with thrombospondin motifs 7; ADAMTS12, A disintegrin and metalloproteinase with thrombospondin motifs 12; COMP, cartilage oligomeric matrix protein; TLR9, Toll-like receptor 9; DR3, death receptor 3; TLIA, TNF-like ligand IA; IL-8, interleukin-8; MS, multiple sclerosis.

Treg cells and production of IL-10 via stimulating phosphorylation of c-Jun N-terminal kinase (JNK). These events depend on TNFR2, but did not rely on PGRNmediated activation of ERK and PI3K pathways. Moreover, forkhead box protein O4/signal transducer and activator of transcription 3 (Foxo4/STAT3) signal pathway are required for PGRN inducted IL-10 generation in Treg cells, particularly in inflammatory arthritis. ${ }^{41,42}$ Also, its anti-inflammatory properties are associated with some chemokines such as CXCL9 and CXCL10. The chemokine network of molecules comprising chemokines and chemokine receptors is central for leukocyte migration and activation with which deregulation may lead to abnormal aggregation of leukocytes in inflammation sites, ultimately resulting in serious inflammatory immune-mediated diseases such as RA and IBD. ${ }^{43-46}$ In addition, PGRN selectively inhibits the expression and releases of TNF- $\alpha$ activated CXCL9 and CXCL10 through TNFR1. Interestingly, PGRN is also available to restrain IFN- $\gamma$ mediated CXCL9 and CXCL10 expression, but this inhibition mechanism is obscure, and more efforts are needed to improve its specific regulatory mechanism. ${ }^{47}$

\section{Induce Chondrogenesis and Cartilage Repair}

In addition to regulating inflammation in immune diseases, another critical protective role of PGRN is as a potent stimulator in response to cartilage differentiation to participate in the development of related immune diseases involving joints or arthritis complications such as RA and T1DM. Recent data show that PGRN is highly increased in the process of cartilage formation 
in vitro and is actively involved in the whole chondrogenesis. ${ }^{48,49}$ The role of PGRN in cartilage development and cartilage repair is regulated by BMP $2 .{ }^{50}$ PGRN reduces cartilage degradation and produces bone protection determined by ERK1/2 signal and its related target gene, especially JunB transcription factor. Moreover, PGRN may inhibit cartilage loss bone resorption mediated by NF- $\kappa B$ pathway in a TNFR1dependent manner. ${ }^{51}$ PGRN is also directly associated with cartilage oligomeric matrix protein (COMP) and chondrocyte proliferation. ${ }^{48}$ COMP is a prominent noncollagenous component of cartilage that exerts a stabilizing effect in the cartilage matrix and degrades severely in joints or arthritis complications. ${ }^{52,53}$ Degradation of COMP evoked by TNF- $\alpha$ may be rescued by PGRN. ${ }^{54}$ PGRN not only inhibits TNF-induced A disintegrin and metalloproteinase with thrombospondin motifs 7/A disintegrin and metalloproteinase with thrombospondin motifs 12 (ADAMTS-7/12) expression, but also interferes with the degradation of COMP by ADAMTS-7/ADAMTS-12, ${ }^{55-57}$ and probably play a central role in the prevention of articular cartilage destruction in arthritis. ${ }^{58}$

\section{Regulation of Toll-Like Receptor 9 (TLR9) Signaling Pathway}

GRN decomposed from PGRN acts as a soluble cofactor in the process of TLR9 signaling induced by oligonucleotide CpG-DNA. ${ }^{59,60}$ Concretely, when PGRN is cleaved into GRN in tissues by elastase, GRN not only acts as a reinforcing agent for a combination between CpG-ODNs and TLR9, but also heavily promotes CpG-ODNs delivery to the localization of TLR9 like endolysosomal compartments. ${ }^{8}$ In addition to the vital role of TLR9 in bacterial defense mentioned above, TLR9 also regulates NF- $\mathrm{kB}$ nuclear translocation ${ }^{61-65}$ which is critical for inflammatory cytokine production and involved in autophagy in many immune-mediated diseases such as RA. ${ }^{66}$ However, whether PGRN may mediate TLR9 signaling pathway in RA has not been reported. What is known at present is PGRN may act as an efficient biological marker for SLE activity and could be active in the pathogenesis and treatment of SLE partly through enhancement of TLR9 signaling. ${ }^{67}$ Further effort is still required to determine more precise mechanisms of the relationship between PGRN and TLR9 in human autoimmune disorders.

\section{PGRN in Immune-Mediated Diseases}

Immune-mediated diseases often result from failing to distinguish self from oneself, ${ }^{68}$ which is characterized by abnormalities in function of immune system and destructed tissues. ${ }^{69}$ Using biological agents to modify specific inflammatory and effector pathways is a promising therapeutic strategy in treating immune-mediated diseases. The agents of blocking TNF- $\alpha$ were approved firstly for therapeutic drugs, and since then drugs for certain autoimmune disorders have been developed. Although the treatment interrupts TNF- $\alpha$ individual continuous progression, the long-term prognoses are not satisfactory for most patients due to its serious side effects. Thus, there is an urgent need to explore novel therapeutic targets and strategies for clinical use. In recent years, emerging evidence has indicated that PGRN may play a role in the etiology and progression of these, including RA, SLE, SSc, IBD, psoriasis, type 1 diabetes mellitus (T1DM) and MS (Table 1).

\section{PGRN in Rheumatoid Arthritis}

RA, the universal autoimmune disease, is caused by persistent inflammation in the synovial joints, which ultimately leads to bone damage as well as disability. ${ }^{1}$ The function of inflammatory cytokines such as TNF- $\alpha$, IL-8, IL- $1 \mathrm{~b}$ and IL-6 in the pathogenesis of RA has been implicated. $^{70,71}$ PGRN is an antagonist of endogenous TNF- $\alpha$, which plays an indispensable role in RA. ${ }^{72,73}$ To examine the exerted effect of endogenous PGRN in the process of inflammation in vivo, researchers pay attention to the investigation of the clinical and histopathological characteristics of $\mathrm{PGRN}^{-/-}$mice model accompanied by collagen-induced arthritis (CIA), and found PGRN null mice are more susceptible to collagen-induced RA which led to a higher RA incidence and more bone and joint destruction than their control littermates, which reverted following the introduction of PGRN.$^{18}$ Additionally, Tang et al. proved that recombinant PGRN administration improved CIA, significantly alleviating the disease severity and inflammatory arthritis mouse models induced by TNF- $\alpha$ transgene. ${ }^{5}$ In RA patients, Chen et al. further showed that the serum PGRN levels were up-regulated. ${ }^{40}$ Meanwhile, Shao et al. suggested that PGRN participates in the regulation of miR-138 down-regulating histone deacetylase 4 (HDAC4) and affect NF-kB level in RA. ${ }^{70}$ Notably, based on the BMP2-induced osteoblast 
Table I Links of PGRN with Several Immune-Mediated Diseases

\begin{tabular}{|c|c|c|c|c|c|}
\hline Diseases & Subjects & Links to PGRN & Year & Author & Ref \\
\hline \multirow[t]{5}{*}{ RA } & $\begin{array}{l}\mathrm{PGRN}^{-1-} \mathrm{C} 57 \mathrm{BL} / 6 \\
\text { mice }\end{array}$ & $\begin{array}{l}\mathrm{PGRN}^{-1-} \text { mice are highly susceptible to CIA, PGRN inhibit TNF- } \alpha- \\
\text { mediated activation of NF-KB and MAPK signaling. }\end{array}$ & 2011 & Tang et al. & [5] \\
\hline & RA patients & $\begin{array}{l}\text { The concentrations of serum PGRN in RA were found to be significantly } \\
\text { higher than control subjects. }\end{array}$ & 2014 & Yamamoto et al. & {$[72]$} \\
\hline & $\mathrm{PGRN}^{-l-}$ B6 mice & $\begin{array}{l}\text { PGRN inhibits expression and release of chemokines CXCL9 and } \\
\text { CXCLI0 in a TNFRI dependent manner. }\end{array}$ & 2016 & Mundra et al. & {$[47]$} \\
\hline & RA patients & Serum PGRN elevated in RA patients, reflecting high disease activity. & 2016 & Chen et al. & {$[40]$} \\
\hline & RA patients & $\begin{array}{l}\text { PGRN rescued miR-I } 38 \text { inhibitor-attenuated inflammatory cytokines } \\
\text { release of FLS cells. }\end{array}$ & 2019 & Shao et al. & {$[70]$} \\
\hline \multirow[t]{3}{*}{ SLE } & SLE patients & PGRN upregulated in serum of SLE, correlated with the serum IL-6 levels. & 2012 & Tanaka et al. & {$[67]$} \\
\hline & SLE patients & $\begin{array}{l}\text { PGRN is up-regulated in the SLE patients and is correlated with pro- } \\
\text { inflammatory cytokines and anti-dsDNA antibody. }\end{array}$ & 2013 & Qiu et al. & {$[76]$} \\
\hline & $\begin{array}{l}\text { SLE Patients, } \\
\text { PGRN }^{-1-} \text { mice }\end{array}$ & $\begin{array}{l}\text { PGRN levels are elevated in SLE patients, PGRN promotes tissue } \\
\text { damage in SLE and exerts proinflammatory functions. }\end{array}$ & 2020 & Jing et al. & {$[12]$} \\
\hline \multirow[t]{4}{*}{ SSc } & LSC patients, & PGRN upregulated in LSC patients. & 2018 & Miyagawa et al. & {$[81]$} \\
\hline & $\begin{array}{l}\text { Human dermal } \\
\text { fibroblasts }\end{array}$ & $\begin{array}{l}\text { PGRN expression regulated by constitutive activation of c-Abl/PKC-d/ } \\
\text { Flil pathway. }\end{array}$ & & & \\
\hline & SSc Patients & $\begin{array}{l}\text { The expression of PGRN was increased in serum from SSc patients and } \\
\text { in skin. }\end{array}$ & 2019 & Yang et al. & {$[77]$} \\
\hline & C57BL/6 mice & $\begin{array}{l}\text { Overexpression of PGRN in SSc stimulates TGF- } \beta / \mathrm{Smad} 3 \text { signaling via } \\
\text { upregulation of T } \beta R \mathrm{RI} \text { mouse model. }\end{array}$ & & & \\
\hline \multirow[t]{2}{*}{ IBD } & $\begin{array}{l}\text { IBD patients, and } \\
\text { mice }\end{array}$ & $\begin{array}{l}\text { Elevated levels of PGRN were found in colitis samples from human IBD } \\
\text { patients and mouse colitis models. }\end{array}$ & 2014 & Wei et al. & [6] \\
\hline & IBD patients & $\begin{array}{l}\text { Upregulated PPGRN-Abs participate in the pathogenesis of the } \\
\text { seropositive subgroup of patients with IBDs by their neutralizing effect } \\
\text { on the plasma level of secreted anti-inflammatory PGRN. }\end{array}$ & 2014 & Thurner et al. & {$[87]$} \\
\hline \multirow[t]{2}{*}{ Psoriasis } & $\begin{array}{l}\text { Psoriasis patients, } \\
\text { PGRN }^{-1-} \text { C57BL/6 } \\
\text { mice }\end{array}$ & $\begin{array}{l}\text { PGRN upregulated in psoriasis serum, PGRN enhanced inflammatory } \\
\text { response to TPA in the skin, decreased percentages of Treg cells. }\end{array}$ & 2015 & Huang et al. & {$[90]$} \\
\hline & $\begin{array}{l}\text { Psoriasis patients, } \\
\text { PGRN }^{-I-} \text { mice }\end{array}$ & $\begin{array}{l}\text { Elevated levels of PGRN were found in colitis samples from human IBD } \\
\text { patients and mouse colitis models in comparison to the corresponding } \\
\text { controls. }\end{array}$ & 2019 & Farag et al. & {$[25]$} \\
\hline MS & MS patients & PGRN is strongly expressed in MS brains. & 2011 & Vercellino et al. & {$[32]$} \\
\hline TIDM & Mice & $\begin{array}{l}\text { PGRN promoted impaired fracture healing in mouse diabetic fracture } \\
\text { models. }\end{array}$ & 2020 & Wei et al. & {$[51]$} \\
\hline
\end{tabular}

Abbreviations: RA, rheumatoid arthritis; SLE, systemic lupus erythematosus; SSc, systemic sclerosis; IBD, inflammatory bowel disease; TIDM, type I diabetes mellitus; MS, multiple sclerosis; PBMC, peripheral blood mononuclear cell; CIA, collagen-induced arthritis; MAPK, mitogen-activated protein kinase; NF- $\mathrm{B}$, nuclear factor of kappa; TNFRI, TNF Receptor I; FLS, Fibroblast-like synoviocytes; anti-dsDNA, anti-double-stranded DNA antibody; LSC, localized scleroderma; PKC-d, protein kinase C-delta; Fli I, Transcription factor Friend leukemia virus integration I; T $\beta$ RI, TGF- $\beta$ receptor I; TPA, I2-O-tetradecanoylphorbol I3-acetate; NF- $k B$, nuclear factor kappa-light-chainenhancer of activated $B$ cells.

differentiation model of mesenchymal stem cell line C2C12, PGRN alleviates the degree of TNF- $\alpha$ preventing osteoblast differentiation and plays a protective role in differentiation of osteoblast under inflammatory conditions. Furthermore, COMP degradation induced by TNF$\alpha$ may be rescued by PGRN. ${ }^{54}$ Altogether, PGRN may 
inhibit TNF-mediated neutrophil activation and cartilage degradation and targeted intervention for PGRN may be an innovative therapy for the treatment of RA in the clinics.

\section{PGRN in Systemic Lupus Erythematosus}

SLE is a prototypic systemic inflammatory autoimmune disease resulting from autoantibodies production, autoreactive $T$ cells that cause tissue, organ damage, and deposition of immune complexes. ${ }^{74,75}$ The serum PGRN of SLE patients was higher compared with that of healthy controls. Serum PGRN levels are considerably associated with the activity of SLE clinical symptoms such as SLE Disease Activity Index and anti-doublestranded DNA antibody (anti-dsDNA) titers, and also correlates inversely with $\mathrm{CH} 50, \mathrm{C} 3$, and $\mathrm{C} 4$ levels which are associated with SLE severity and kidney injury. Moreover, the level of serum PGRN was markedly decreased following the successful SLE treatment. ${ }^{67}$ Furthermore, increased PGRN levels were positively correlated with the severity of pristane-induced murine model of SLE. Compared with WT SLE mice, tissue injuries significantly alleviated and autoantibody presence including anti-dsDNA and anti-ribosomal protein P0 basically reduced in PGRN-deficient $\left(\mathrm{PGRN}^{-1}\right) \mathrm{SLE}$ mice. It is worth noting that destroyed $\mathrm{T}$ cell homeostasis is regarded as the significant pathological effect of SLE, associated studies have shown that PGRN could regulate immune responses of $\mathrm{T}$ lymphocyte mediation, because PGRN $^{-/-}$SLE mice have lower levels of Th1 and Th17 levels and higher levels of Th2 and Treg cells than WT mice in the spleens, leading to inflammatory cytokines significantly decreasing such as Interferon gamma (IFN$\gamma$ ) and interleukin-17A (IL-17A) and anti-inflammatory cytokines of IL-4 and IL-10 increasing. ${ }^{12}$ However, the above conclusion is contrary to the fact that PGRN can promote the differentiation of CD4+T cells into Tregs. The contradictory findings imply that the manner of PGRN regulating $T$ cell functions differs in diverse disease states. Nevertheless, further studies are needed to examine the exact role regulated by PGRN in SLE.

Mechanically, PGRN might be an efficient disease activity index participating in the SLE pathogenesis partly by reinforcing the TLR9 signaling and the serum PGRN levels evidently and correlating with the serum IL-6 expression; CpG-B stimulated the PBMCs products of IL-6, which was upregulated by application of the recombinant human progranulin (rhPGRN). ${ }^{67}$ From the therapeutic perspective, it has been shown that the
PGRN levels decreased after administration of large doses of prednisone. Prednisone is one of the glucocorticoids (GCs) and is generally acknowledged as an antiinflammatory immunosuppressant. ${ }^{76}$ Therefore, it is not possible to fully establish that PGRN promotes the development of SLE based on the existing literature, and further studies are needed to refine the specific mechanism of PGRN on this disease.

\section{PGRN in Systemic Sclerosis}

SSc, also known as scleroderma, is an autoimmune fibrotic disease featured with the loss of control and sustained activation of fibroblasts, leading to excessive accumulation of collagen mainly including collagen type I (COL1) and (COL3), skin thickening, and differentiation of fibroblasts into myofibroblasts following an increase in $\alpha$-smooth muscle actin ( $\alpha$-SMA). ${ }^{77-79}$ SSc loosely consists of two entirely different clinical classifications: systemic sclerosis (SSc) and localized scleroderma (LSC). ${ }^{80}$ Present literature has observed a distinct increment in PGRN in vivo and in vitro. Yang et al. have discovered a significant increment in the serum PGRN level in SSc patients. Results from immunohistology and RT-qPCR displayed that mRNA and protein of PGRN was also significantly elevated in the lesional skin from the scleroderma mouse model of BLM-induced manner. At the same time, their experiment on $\mathrm{PGRN}^{-/}$mice further showed both attenuation of dermal fibrosis and amelioration of lesion myofibroblasts differentiation. Downregulation of TGF- $\beta$ receptor $I(T \beta R I)$ and $p-S m a d 3$ may account for the reduced severity degree of skin sclerosis in $\mathrm{PGRN}^{-1-}$ mice, following corresponding diminution of connective tissue growth factor (CTGF), which is a downstream target gene of p-Smad3 in skin lesion. Conversely, impaired T $\beta R$ I and p-Smad3 level could be significantly reversed by exogenous PGRN in mice dermal fibroblasts. ${ }^{77}$

Additionally, in BLM-treated mice, Miyagawa et al. indicated that LSC dermal fibroblasts shared the Transcription factor Friend leukemia virus integration 1 (Fli1) deficiency and PGRN-dependent profibrotic actions with SSc dermal fibroblasts. ${ }^{81}$ Also, deleted Fli-1 transcription factor in SSc was found to cause the increase of PGRN expression through directly binding to the GRN promoter region to repress PGRN production. ${ }^{18}$ In vitro, the activated $\mathrm{c}-\mathrm{Abl} /$ protein kinase $\mathrm{C}-\delta /$ Fli1 (c-Abl/PKC-d/Fli1) pathway partly results in PGRN overproduction, which might help dermal fibroblasts counteract the partial anti-fibrotic effect of TNF- $\alpha$. Given that the TNF- $\alpha$ roles in fibrosis are 
a double-edged sword in vitro and in vivo, ${ }^{82-84}$ it is not possible to confirm whether PGRN displayed the similarity function and further investigations are needed to explore the exact molecular mechanisms of PGRN in SSc.

\section{PGRN in Inflammatory Bowel Disease}

IBD often presents as chronic relapsing intestinal inflammation, ${ }^{85}$ which encompasses two distinct diseases according to the clinical, histopathological, and pathogenetic characteristics, namely ulcerative colitis (UC) and Crohn's disease (CD). ${ }^{86}$ In both diseases, innate and adaptive immune function changes are ultimately accompanied by increase of proinflammatory cytokine levels such as TNF- $\alpha$, IL-6, IL- $1 b .{ }^{87}$ Though the specific etiology of IBD remains unclear, with our understanding of IBD advancing in recent years, it has been shown that PGRN exerts a crucial role in mucosal inflammatory response and damage, and its effect has been confirmed in both IBD patients and mouse experimental colitis models. Wei et al. tested $\mathrm{PGRN}^{-/-}$mice and saw that they developed more severe colitis in comparison to the wild-control mice in the colitis model induced by chemical material such as dextran sulfate sodium (DSS) and trinitrobenzene sulfonic acid (TNBS). Also, lack of PGRN signaling in CD41 T cells intensifies experimental colitis. However, recombinant PGRN improves colitis syndrome. In addition, TNFR2 and IL-10 signaling are crucial to protective action of PGRN in IBD. ${ }^{6}$ Similarly, in addition to PGRN, neutralizing autoantibodies against PGRN (PGRN-Abs) also act on the serum of patients with multiple autoimmune disorders, particularly IBD. PGRN-Abs detected in $16.31 \%$ of CD patients and in $21.13 \%$ of UC patients demonstrated considerable effects of neutralizing PGRN levels in plasma. PGRN-Abs also showed a pro-inflammatory effect in IBD. ${ }^{18}$ Collectively, these studies show that PGRN is a crucial mediator of intestinal homeostasis, which dysregulated will aggravate intestinal inflammation during colitis. The protective action of PGRN in IBD principally relies on the TNFR2-mediated IL-10 signaling pathway. These findings describe not only the significant antiinflammatory role of PGRN in vivo but also provide a novel strategy for PGRN and its derivatives in the treatment of intestinal inflammation ${ }^{6}$

\section{PGRN in Psoriasis}

Psoriasis is a genetic immune-mediated inflammatory skin disease that is often accompanied by hyperproliferation of the epidermal skin and a change of various vascular properties in the dermal layer. ${ }^{88,89}$ Increasing evidence has indicated that PGRN plays a critical role in psoriasis. Serum PGRN levels are dramatically incremented in psoriasis patients and PGRN were also found to be significantly increased in the psoriasis-like lesions of 12O-tetradecanoylphorbol 13-acetate (TPA)-treated WT mice. ${ }^{25}$ Surprisingly, PGRN $^{-/-}$mice were sensitive to TPA-induced psoriasis-like inflammation. PGRN may protect the skin from the exaggerated psoriasis-like inflammatory response by promoting Treg cells differentiating and recruiting at the site of inflammation..$^{90}$ Additionally, Tian et al. investigated that PGRN was evidently expressed highly in inflammatory $\mathrm{HaCaT}$ cells and overexpression of PGRN inhibits the TNF- $\alpha$-induced inflammation in keratinocytes and negatively regulates the production of inflammatory factors interleukin-1beta (IL$1 \beta$ ), interleukin 6 (IL-6), cyclooxygenase-2 (COX-2), inducible nitric oxide synthase (iNOs), and monocyte chemoattractant protein-1 (MCP-1) and positively mediates autophagy through the $\mathrm{Wnt} / \beta$-catenin signaling pathway. ${ }^{88}$

Although PGRN overexpression may be used as a potential immunotherapeutic option for psoriasis management programs, there are also studies suggesting that PGRN is considered as a pro-inflammatory cytokine in the pathogenesis of psoriasis. In the first instance, serum PGRN/TNF- $\alpha$ ratio was found to be negatively correlated with disease severity and also, Farag et al. identified that PGRN shifted from the circulation to the skin may be involved in the inflammatory response of keratinocytes at the tissue level in psoriasis by reducing the expression of $\beta$-catenin in psoriasis. This discrepancy may be attributed to the use of different methods. Some focused on the correlation of PGRN serum level, others depend on the expression of PGRN tissue. ${ }^{25}$

\section{Other Immune-Mediated Diseases}

Apart from the disorders mentioned in the above immunemediated diseases, PGRN has also been proved to be actively involved in the etiology of several other immunemediated diseases, such as T1DM and MS and to play a protective role in both diseases.

T1DM is a major subtype of diabetes, which features islet $\beta$ cells being destroyed in the pancreas caused by factors of heredity and environment, ${ }^{91}$ and patients with diabetes are often accompanied by an evidently increased risk of fracture and poor fracture healing. Studies on the role of PGRN in T1MD mainly focused on the diabetic bone healing process. Wei et al. employed multiple models 
of diabetic fracture and found that the $\mathrm{PGRN}^{-1-}$ mice showed more severe delayed diabetic bone healing than controls. They also utilized recombinant PGRN, which may also promote diabetic fracture healing through accelerating callus formation and transformation of cartilage to bone. Under the diabetic condition, PGRN not only reduced the transcriptional levels of pro-inflammatory markers, including IL-1 $\beta$, nitric oxide synthase-2 (NOS2), and COX-2 but also significantly promoted the levels of chondrogenic marker genes such as type II collagen and aggrecan. Mechanically, the effects of PGRN in promoting the diabetic fracture healing is possibly through binding to TNFR1 to inhibit TNF- $\alpha$-mediated inflammatory and bone erosion, and activating the Akt, ERK1/2, and mTOR signaling to accelerate formation of callus and promotion of callus transfer into new bone. ${ }^{51}$

With regards to $\mathrm{MS}$, it is a chronic, predominantly autoimmune disease characterized by injury of the central nervous system (CNS), frequently resulting in neurological disability in young adults globally. ${ }^{92,93}$ PGRN is highly expressed in active demyelinating lesions by macrophages/ microglia and in the normal appearing white matter (NAWM) by activated microglia; concentrations of MS PGRN cerebrospinal fluid (CSF) are correspondingly elevated in the case of activation of enhanced macrophage/ microglia during progressive and recurrent MS. PGRN might protect against neuroinflammation in MS brains, downregulating the degree of injury of diverse neuronal cell populations induced by noxious stimuli. ${ }^{94}$ However, elevated CSF concentration of PGRN seems not specific to MS, but a common feature of CNS inflammation.

\section{PGRN as a Promising Therapeutic Target for Immune-Mediated Diseases}

PGRN, a double-edged sword, plays a pro-inflammatory or anti-inflammatory role in response to the different characteristics of its immune system diseases and targeted PGRN has promise as a potential approach for immunemediated diseases. Understanding the intrinsic mechanisms driving PGRN and striving to find and develop reagents that specifically stimulate PGRN activation, inhibit its fragmentation or development of PGRN derivatives, may open an avenue to novel therapeutic options for immune-mediated diseases (Figure 1). ${ }^{9}$

Few studies have been done on several immune-mediated diseases by affecting the activity of PGRN. Understanding the molecules or pathways required for PGRN activation/inhibition is the first step in finding possible intervention targets. Given posttranslational modification, as a novel posttranscriptional regulator in mediating PGRN expression, microRNA-29b (miR-29b) binds directly to the human PGRN (hPGRN) to regulate the endogenous hPGRN expression through its $3^{\prime}$ untranslated region (3'UTR). ${ }^{95}$ Moreover, miR-29b-3p (3p means 3 prime end of the hairpin) facilitates chondrocyte apoptosis and aggravates the process of osteoarthritis by inhibiting PGRN. ${ }^{96}$ MiR-34b-5p inhibition has to be proved to activate PGRN that ameliorates inflammation. ${ }^{97}$ miR-138 activates PGRN to promote RA via regulating HDAC4. ${ }^{70}$ Similarly, down-regulation of sortilin 1 (SORT1) expression is a pivotal mechanism of PGRN elevation and a prospective target for PGRN to promote arthritis and other diseases, as indicated by PGRN KO mice phenotype. ${ }^{98}$ As for the protection of PGRN from proteolytic degradation, some proteins, such as the secretory leukocyte protease inhibitor (SLPI) and apolipoprotein A1 can bind to it to protect it against proteolytic degradation. ${ }^{34}$ Besides, an engineered protein consisted of the FAC domain of PGRN was constructed, which retains comparable TNFR binding affinity of PGRN referred as Atsttrin. More importantly, Atsttrin surpasses PGRN in delaying the degree of inflammation and in reducing mice susceptibility to collagen-induced arthritis RA in vivo, ${ }^{5}$ and Atsttrin-mediated mesenchymal stem cells joint therapy guard against osteoarthritis progress in a surgically induced murine osteoarthritis model. ${ }^{99}$ Additionally, Atsttrin lacks the carcinogenic activity of PGRN, ${ }^{100,101}$ which has attracted more attention, and the composition of Atsttrin prevents it from being digested by a single particle unit. Even at extremely high doses, no cytotoxicity or lethality associated with Atsttrin was observed. In terms of pharmacokinetics, Atsttrin is well absorbed after intraperitoneal administration, showing high stability, and its half-life is significantly longer compared with PGRN, so the application of Atsttrin gets more and more attention. Recombinant PGRN can also reduce the severity of the disease in collagen antibody induction, collagen induction, and TNF- $\alpha$ transgenic inflammatory arthritis mouse models. Collectively, studying the mechanism of PGRN in immune-mediated diseases and striving to develop specific PGRN-activated agents may provide directions for the development of drugs for immune-mediated diseases.

\section{Conclusion}

A solid basis of data from in vitro and in vivo models is accumulating to clarify the molecular mechanisms of PGRN in autoimmune disease. Particularly, the regulation of TNF- $\alpha$ / 
TNFR by PGRN is seen to have been characterized in detail, and the targeted PGRN may have some advantages compared with the existing TNF- $\alpha$ inhibitors. However, much remains to be explored about unresolved questions of PGRN in immunemediated diseases. Firstly, the PGRN plays an essential role in immune-mediated diseases mainly as an extracellular protein and mediates the folding and transportation of its multiple binding partners in neurological diseases mainly as an intracellular protein. For example, PGRN binds to the soluble lysosomal protein, particularly Prosaposin (PSAP), participating in neurological disorders such as frontotemporal lobar degeneration (FTLD). However, extracellular PGRN binding to membrane receptors mediates PGRN uptake and regulates intracellular PGRN levels; whether intracellular PGRN participates in the occurrence of immune diseases and extracellular and intracellular PGRN exert functions that are integrated or completely independent remain to be clarified. Secondly, many studies have investigated the expression of PGRN in immunemediated diseases, but the results are contradictory or inconclusive. Finally, in addition to acting on TNFR, TLR9, and DR3 receptors, PGRN has also been shown to be involved in the regulation of the Notch receptor ${ }^{102}$ and Eph receptor A2 (EphA2), ${ }^{103}$ which also affect the function of the immune system, such as Notch1-4 is abnormally expressed in RA synovial cells, NF- $\mathrm{kB}$-related pro-inflammatory activities in SLE M2b macrophages partly stimulated by Notch1 and EphA2 is a potential target for RA. ${ }^{104-106}$ Whether the relationship is indirect or direct between Notch/EphA2 and PGRN remains to be determined. In recent years, there is growing interest in the efforts to develop PGRN targeting treatments to overcome the current bottlenecks and also an urgent need to explore more precise actions of PGRN and GRN, especially in human systems and in host defense in order to comprehensively explore the role of PGRN in immune-mediated diseases.

\section{Abbreviations}

PGRN, Progranulin; RA, rheumatoid arthritis; SLE, systemic lupus erythematosus; SSc, systemic sclerosis; IBD, inflammatory bowel disease; T1DM, type 1 diabetes mellitus; MS, multiple sclerosis; TNF- $\alpha$, tumor necrosis factor alpha; TNFR, TNF receptor; TNFR1, TNF Receptor 1; Treg, regulatory T cells; Th1, T-helper 1; Th17, T-helper 17; JAK, Janus kinase; STAT, signal transducer and activator of transcription; CpG-ODNs, CpG oligonucleotides; TLR9, Toll-like receptor 9; PEPI, proepithelin; PCDGF, PC-cell-derived growth factor; GEP, Granulin-epithelin precursor; MMP-9, matrix metalloproteinase-9; PRTN3, proteinase 3; CRD2, cysteine-rich domain 2; ERK1/2, extracellular regulated protein kinase 1 and 2; PI3K, phosphatidylinositol 3 kinases; AKT, protein kinase B; NF-kB; nuclear factor of kappa; DR3, death receptor 3; TL1A, TNF-like ligand 1A; JNK, c-Jun N-terminal kinase; FOXO4, forkhead box protein O4; STAT3, signal transducer and activator of transcription 3; COMP, cartilage oligomeric matrix protein; ADAMTS-7, A disintegrin and metalloproteinase with thrombospondin motifs 7; FLS, Fibroblast-like synoviocytes; anti-dsDNA, anti-double-stranded DNA antibody; LSC, localized scleroderma; Fli1, Transcription factor Friend leukemia virus integration 1 ; T $\beta R I$, TGF- $\beta$ receptor I; TPA, 12 O-tetradecanoylphorbol 13-acetate; IFN- $\gamma$, Interferon gamma; IL-17A, interleukin-17A; rhPGRN, recombinant human progranulin; GCs, glucocorticoids; COL1, collagen type I; $\alpha$ SMA, $\alpha$-smooth muscle actin; T $\beta R I$, TGF- $\beta$ receptor I; CTGF, connective tissue growth factor; Fli1, Transcription factor Friend leukemia virus integration 1; DSS, dextran sulfate sodium; TNBS, trinitrobenzene sulfonic acid; PGRN-Abs, neutralizing autoantibodies against PGRN; IL-1 $\beta$, interleukin1beta; IL-6, Interleukin 6; COX-2, cyclooxygenase-2; iNOs, Inducible nitric oxide synthase; MCP-1, Monocyte chemoattractant protein-1; NOS2, nitric oxide synthase-2; CNS, central nervous system; NAWM, normal appearing white matter; CSF, cerebrospinal fluid; SORT1, sortilin 1; SLPI, secretory leukocyte protease inhibitor. PSAP, Prosaposin; FTLD, frontotemporal lobar degeneration.

\section{Acknowledgments}

This research was supported by the National Natural Science Foundation of China. (No. 81274172, 81473267 and 81973637); The National Traditional Chinese Medicine Inheritance and Innovation "Hundreds and Thousands" Talent Project: Young Qihuang Scholar Support Project of the State Administration of Traditional Chinese Medicine in 2020.

\section{Disclosure}

The authors report no conflicts of interest in this work.

\section{References}

1. Tao JH, Cheng M, Tang JP, Liu Q, Pan F, Li XP. Foxp3, regulatory $\mathrm{T}$ cell, and autoimmune diseases. Inflammation. 2017;40(1):328-339. doi:10.1007/s10753-016-0470-8

2. Abdolmaleki F, Kovanen PT, Mardani R, Gheibi-Hayat SM, Bo S, Sahebkar A. Resolvins: emerging players in autoimmune and inflammatory diseases. Clin Rev Allergy Immunol. 2020;58(1):82-91. doi:10.1007/s12016-019-08754-9

3. Jian J, Konopka J, Liu C. Insights into the role of progranulin in immunity, infection, and inflammation. J Leukoc Biol. 2013;93 (2):199-208. doi:10.1189/jlb.0812429 
4. Wei F, Zhang Y, Zhao W, Yu X, Liu CJ. Progranulin facilitates conversion and function of regulatory $\mathrm{T}$ cells under inflammatory conditions. PLoS One. 2014;9(11):e112110. doi:10.1371/journal. pone. 0112110

5. Tang W, Lu Y, Tian QY, et al. The growth factor progranulin binds to TNF receptors and is therapeutic against inflammatory arthritis in mice. Science. 2011;332(6028):478-484. doi:10.1126/ science. 1199214

6. Wei F, Zhang Y, Jian J, et al. PGRN protects against colitis progression in mice in an IL-10 and TNFR2 dependent manner. Sci Rep. 2014;4:7023. doi:10.1038/srep07023

7. Li L, Li L, Xiao L, Shangguan J. Progranulin ameliorates coxsackievirus-B3-induced viral myocarditis by downregulating Th1 and Th17 cells. Exp Cell Res. 2018;367(2):241-250. doi:10.1016/j.yexcr.2018.04.001

8. Park B, Buti L, Lee S, et al. Granulin is a soluble cofactor for toll-like receptor 9 signaling. Immunity. 2011;34(4):505-513. doi:10.1016/j.immuni.2011.01.018

9. Shen HH, Yang YX, Meng X, et al. NLRP3: a promising therapeutic target for autoimmune diseases. Autoimmun Rev. 2018;17 (7):694-702. doi:10.1016/j.autrev.2018.01.020

10. Moresco EM, Beutler B. Special delivery: granulin brings $\mathrm{CpG}$ DNA to Toll-like receptor 9. Immunity. 2011;34(4):453-455. doi:10.1016/j.immuni.2011.04.001

11. Cui Y, Hettinghouse A, Liu CJ. Progranulin: a conductor of receptors orchestra, a chaperone of lysosomal enzymes and a therapeutic target for multiple diseases. Cytokine Growth Factor Rev. 2019;45:53-64. doi:10.1016/j.cytogfr.20 19.01 .002

12. Jing C, Zhang X, Song Z, Zheng Y, Yin Y. Progranulin Mediates Proinflammatory Responses in Systemic Lupus Erythematosus: implications for the Pathogenesis of Systemic Lupus Erythematosus. J Interferon Cytokine Res. 2020;40(1):33-42. doi:10.1089/jir.2019.0047

13. Bateman A, Cheung ST, Bennett HPJ, Brief A. Overview of Progranulin in Health and Disease. Methods Mol Biol. 2018;1806:3-15.

14. Bhandari V, Palfree RG, Bateman A. Isolation and sequence of the granulin precursor cDNA from human bone marrow reveals tandem cysteine-rich granulin domains. Proc Natl Acad Sci U S A. 1992;89(5):1715-1719. doi:10.1073/pnas.89. 5.1715

15. Hrabal R, Chen Z, James S, Bennett HP, Ni F. The hairpin stack fold, a novel protein architecture for a new family of protein growth factors. Nat Struct Biol. 1996;3(9):747-752. doi:10. 1038/nsb0996-747

16. Palfree RG, Bennett HP, Bateman A. The Evolution of the Secreted Regulatory Protein Progranulin. PLoS One. 2015;10(8) e0133749. doi:10.1371/journal.pone.0133749

17. Plowman GD, Green JM, Neubauer MG, et al. The epithelin precursor encodes two proteins with opposing activities on epithelial cell growth. J Biol Chem. 1992;267(18):13073-13078. doi:10.1016/S0021-9258(18)42382-4

18. Jian J, Li G, Hettinghouse A, Liu C. Progranulin: a key player in autoimmune diseases. Cytokine. 2018;101:48-55. doi:10.1016/j. cyto.2016.08.007

19. Daniel R, He Z, Carmichael KP, Halper J, Bateman A. Cellular localization of gene expression for progranulin. $J$ Histochem Cytochem. 2000;48(7):999-1009. doi:10.1177/002215540004800713

20. Petkau TL, Leavitt BR. Progranulin in neurodegenerative disease. Trends Neurosci. 2014;37(7):388-398. doi:10.1016/j.tins.2014. 04.003

21. Matsubara T, Mita A, Minami K, et al. PGRN is a key adipokine mediating high fat diet-induced insulin resistance and obesity through IL-6 in adipose tissue. Cell Metab. 2012;15(1):38-50. doi:10.1016/j.cmet.2011.12.002
22. Abella V, Scotece M, Conde J, et al. The novel adipokine progranulin counteracts IL-1 and TLR4-driven inflammatory response in human and murine chondrocytes via TNFR1. Sci Rep. 2016;6:20356. doi:10.1038/srep20356

23. Pan CX, Kinch MS, Kiener PA, et al. PC cell-derived growth factor expression in prostatic intraepithelial neoplasia and prostatic adenocarcinoma. Clin Cancer Res. 2004;10(4):1333-1337. doi:10.1158/1078-0432.CCR-1123-03

24. Cheung PF, Cheng CK, Wong NC, et al. Granulin-epithelin precursor is an oncofetal protein defining hepatic cancer stem cells. PLoS One. 2011;6(12):e28246. doi:10.1371/journal.pone.0028 246

25. Farag AGA, Shoaib MA, Samaka RM, Abdou AG, Mandour MM, Ibrahim RAL. Progranulin and beta-catenin in psoriasis: an immunohistochemical study. J Cosmet Dermatol. 2019;18(6):2019-2026. doi:10.1111/jocd.12966

26. $\mathrm{Xu} \mathrm{D,} \mathrm{Suenaga} \mathrm{N,} \mathrm{Edelmann} \mathrm{MJ,} \mathrm{Fridman} \mathrm{R,} \mathrm{Muschel} \mathrm{RJ,}$ Kessler BM. Novel MMP-9 substrates in cancer cells revealed by a label-free quantitative proteomics approach. Mol Cell Proteomics. 2008;7(11):2215-2228. doi:10.1074/mcp.M800095MCP200

27. Suh HS, Choi N, Tarassishin L, Lee SC. Regulation of progranulin expression in human microglia and proteolysis of progranulin by matrix metalloproteinase-12 (MMP-12). PLoS One. 2012;7(4): e35115. doi:10.1371/journal.pone.0035115

28. Butler GS, Dean RA, Tam EM, Overall CM. Pharmacoproteomics of a metalloproteinase hydroxamate inhibitor in breast cancer cells: dynamics of membrane type 1 matrix metalloproteinase-mediated membrane protein shedding. $\mathrm{Mol}$ Cell Biol. 2008;28(15):4896-4914. doi:10.1128/MCB.01775-07

29. Ungurs MJ, Sinden NJ, Stockley RA. Progranulin is a substrate for neutrophil-elastase and proteinase- 3 in the airway and its concentration correlates with mediators of airway inflammation in COPD. Am J Physiol Lung Cell Mol Physiol. 2014;306(1): L80-87. doi:10.1152/ajplung.00221.2013

30. Kessenbrock K, Frohlich L, Sixt M, et al. Proteinase 3 and neutrophil elastase enhance inflammation in mice by inactivating antiinflammatory progranulin. J Clin Invest. 2008;118(7):24 38-2447. doi:10.1172/JCI34694

31. Zhu J, Nathan C, Jin W, et al. Conversion of proepithelin to epithelins: roles of SLPI and elastase in host defense and wound repair. Cell. 2002;111(6):867-878. doi:10.1016/S0092-8674(02)01141-8

32. Vercellino M, Grifoni S, Romagnolo A, et al. Progranulin expression in brain tissue and cerebrospinal fluid levels in multiple sclerosis. Mult Scler. 2011;17(10):1194-1201. doi:10.1177/ 1352458511406164

33. Samejima T, Nagamatsu T, Akiba N, et al. Secretory leukocyte protease inhibitor and progranulin as possible regulators of cervical remodeling in pregnancy. $J$ Reprod Immunol. 2021;14 3:103241. doi:10.1016/j.jri.2020.103241

34. Okura H, Yamashita S, Ohama T, et al. HDL/apolipoprotein A-I binds to macrophage-derived progranulin and suppresses its conversion into proinflammatory granulins. $J$ Atheroscler Thromb. 2010;17(6):568-577. doi:10.5551/jat.3921

35. Liu CJ. Progranulin: a promising therapeutic target for rheumatoid arthritis. FEBS Lett. 2011;585(23):3675-3680. doi:10.1016/j. febslet.2011.04.065

36. Tian Q, Zhao Y, Mundra JJ, et al. Three TNFR-binding domains of PGRN act independently in inhibition of TNF-alpha binding and activity. Front Biosci. 2014;19:1176-1185. doi:10.2741/4274

37. Tian Q, Zhao S, Liu C. A solid-phase assay for studying direct binding of progranulin to TNFR and progranulin antagonism of TNF/TNFR interactions. Methods Mol Biol. 2014;1155:163-172.

38. Thurner L, Fadle N, Regitz E, et al. The molecular basis for development of proinflammatory autoantibodies to progranulin. J Autoimmun. 2015;61:17-28. doi:10.1016/j.jaut.2015.05.002 
39. Liu C, Li XX, Gao W, Liu W, Liu DS. Progranulin-derived Atsttrin directly binds to TNFRSF25 (DR3) and inhibits TNF-like ligand 1A (TL1A) activity. PLoS One. 2014;9(3): e92743. doi:10.1371/journal.pone. 0092743

40. Chen J, Li S, Shi J, et al. Serum progranulin irrelated with Breg cell levels, but elevated in RA patients, reflecting high disease activity. Rheumatol Int. 2016;36(3):359-364. doi:10.1007/s002 96-015-3372-4

41. Tian G, Jin X, Wang Q, Ye T, Li G, Liu J. Recent advances in the study of progranulin and its role in sepsis. Int Immunopharmacol. 2020;79:106090. doi:10.1016/j.intimp.2019.106090

42. Fu W, Hu W, Shi L, et al. Foxo4- and Stat3-dependent IL-10 production by progranulin in regulatory $\mathrm{T}$ cells restrains inflammatory arthritis. FASEB J. 2017;31(4):1354-1367. doi:10.1096/ fj.201601134R

43. Johnson Z, Power CA, Weiss $\mathrm{C}$, et al. Chemokine inhibition-why, when, where, which and how? Biochem Soc Trans. 2004;32(Pt 2):366-377. doi:10.1042/bst0320366

44. Kuan WP, Tam LS, Wong CK, et al. CXCL 9 and CXCL 10 as Sensitive markers of disease activity in patients with rheumatoid arthritis. J Rheumatol. 2010;37(2):257-264. doi:10.3899/jrheum. 090769

45. Lee EY, Lee ZH, Song YW. The interaction between CXCL10 and cytokines in chronic inflammatory arthritis. Autoimmun Rev. 2013;12(5):554-557. doi:10.1016/j.autrev.2012.10.001

46. Ruschpler P, Lorenz P, Eichler W, et al. High CXCR3 expression in synovial mast cells associated with CXCL9 and CXCL10 expression in inflammatory synovial tissues of patients with rheumatoid arthritis. Arthritis Res Ther. 2003;5(5):R241-252. doi:10. $1186 / \mathrm{ar} 783$

47. Mundra JJ, Jian J, Bhagat P, Liu CJ. Progranulin inhibits expression and release of chemokines CXCL9 and CXCL10 in a TNFR1 dependent manner. Sci Rep. 2016;6:21115. doi:10. 1038/srep21115

48. Xu K, Zhang Y, Ilalov $\mathrm{K}$, et al. Cartilage oligomeric matrix protein associates with granulin-epithelin precursor (GEP) and potentiates GEP-stimulated chondrocyte proliferation. $J$ Biol Chem. 2007;282(15):11347-11355. doi:10.1074/jbc.M608744200

49. Feng JQ, Guo FJ, Jiang BC, et al. Granulin epithelin precursor: a bone morphogenic protein 2-inducible growth factor that activates Erk1/2 signaling and JunB transcription factor in chondrogenesis. FASEB J. 2010;24(6):1879-1892. doi:10.1096/ fj. 09-144659

50. Zhao YP, Tian QY, Frenkel S, Liu CJ. The promotion of bone healing by progranulin, a downstream molecule of BMP-2, through interacting with TNF/TNFR signaling. Biomaterials. 2013;34(27):6412-6421. doi:10.1016/j.biomaterials.2013.05.030

51. Wei J, Zhang L, Ding Y, et al. Progranulin promotes diabetic fracture healing in mice with type 1 diabetes. Ann N Y Acad Sci. 2020;1460(1):43-56. doi:10.1111/nyas.14208

52. Turesson C, Bergstrom U, Jacobsson LT, Truedsson L, Berglund G, Saxne T. Increased cartilage turnover and circulating autoantibodies in different subsets before the clinical onset of rheumatoid arthritis. Ann Rheum Dis. 2011;70(3):520-522. doi:10.1136/ard.2010.131896

53. Posey KL, Coustry F, Hecht JT. Cartilage oligomeric matrix protein: cOMPopathies and beyond. Matrix Biol. 2018;7172:161-173. doi:10.1016/j.matbio.2018.02.023

54. Wang N, Zhang J, Yang JX. Growth factor progranulin blocks tumor necrosis factor-alpha-mediated inhibition of osteoblast differentiation. Genet Mol Res. 2016;15(3):215.

55. Neidhart M, Hauser N, Paulsson M, DiCesare PE, Michel BA, Hauselmann HJ. Small fragments of cartilage oligomeric matrix protein in synovial fluid and serum as markers for cartilage degradation. Br J Rheumatol. 1997;36(11):1151-1160. doi:10. 1093/rheumatology/36.11.1151
56. Liu CJ, Kong W, Ilalov $\mathrm{K}$, et al. ADAMTS-7: a metalloproteinase that directly binds to and degrades cartilage oligomeric matrix protein. FASEB J. 2006;20(7):988-990. doi:10.1096/fj.05-3877fje

57. Liu CJ, Kong W, Xu K, et al. ADAMTS-12 associates with and degrades cartilage oligomeric matrix protein. $J$ Biol Chem. 2006;281(23):15800-15808. doi:10.1074/jbc.M513433200

58. Guo F, Lai Y, Tian Q, Lin EA, Kong L, Liu C. Granulin-epithelin precursor binds directly to ADAMTS-7 and ADAMTS-12 and inhibits their degradation of cartilage oligomeric matrix protein. Arthritis Rheum. 2010;62(7):2023-2036. doi:10.1002/art.27491

59. Hemmi H, Takeuchi O, Kawai T, et al. A Toll-like receptor recognizes bacterial DNA. Nature. 2000;408(6813):740-745. doi: $10.1038 / 35047123$

60. Krieg AM. CpG motifs in bacterial DNA and their immune effects. Аnпu Rev Immunol. 2002;20:709-760. doi:10.1146/ annurev.immunol.20.100301.064842

61. Latz E, Verma A, Visintin A, et al. Ligand-induced conformational changes allosterically activate Toll-like receptor 9. Nat Immunol. 2007;8(7):772-779. doi:10.1038/ni1479

62. Kim YM, Brinkmann MM, Paquet ME, Ploegh HL. UNC93B1 delivers nucleotide-sensing toll-like receptors to endolysosomes. Nature. 2008;452(7184):234-238. doi:10.1038/nature06726

63. Ewald SE, Lee BL, Lau L, et al. The ectodomain of Toll-like receptor 9 is cleaved to generate a functional receptor. Nature. 2008;456(7222):658-662. doi:10.1038/nature07405

64. Avalos AM, Kirak O, Oelkers JM, et al. Cell-specific TLR9 trafficking in primary APCs of transgenic TLR9-GFP mice. J Immunol. 2013;190(2):695-702. doi:10.4049/jimmunol.1202 342

65. Latz E, Schoenemeyer A, Visintin A, et al. TLR9 signals after translocating from the ER to $\mathrm{CpG}$ DNA in the lysosome. Nat Immunol. 2004;5(2):190-198. doi:10.1038/ni1028

66. Wei W, Ren J, Yin W, et al. Inhibition of Ctsk modulates periodontitis with arthritis via downregulation of TLR9 and autophagy. Cell Prolif. 2020;53(1):e12722. doi:10.1111/cpr.12722

67. Tanaka A, Tsukamoto $\mathrm{H}$, Mitoma $\mathrm{H}$, et al. Serum progranulin levels are elevated in patients with systemic lupus erythematosus, reflecting disease activity. Arthritis Res Ther. 2012;14(6):R244. doi:10.1186/ar4087

68. Wu GC, Pan HF, Leng RX, et al. Emerging role of long noncoding RNAs in autoimmune diseases. Autoimmun Rev. 2015;14 (9):798-805. doi:10.1016/j.autrev.2015.05.004

69. Pan HF, Li XP, Zheng SG, Ye DQ. Emerging role of interleukin-22 in autoimmune diseases. Cytokine Growth Factor Rev. 2013;24(1):51-57. doi:10.1016/j.cytogfr.2012.07.002

70. Shao L, Hou C. miR-138 activates NF-kappaB signaling and PGRN to promote rheumatoid arthritis via regulating HDAC4. Biochem Biophys Res Commun. 2019;519(1):166-171. doi:10. 1016/j.bbrc.2019.08.092

71. Atzeni F, Nucera V, Masala IF, Sarzi-Puttini P, Bonitta G. Il-6 Involvement in pain, fatigue and mood disorders in rheumatoid arthritis and the effects of Il-6 inhibitor sarilumab. Pharmacol Res. 2019;149:104402. doi:10.1016/j.phrs.2019.104402

72. Yamamoto Y, Takemura M, Serrero G, et al. Increased serum GP88 (Progranulin) concentrations in rheumatoid arthritis. Inflammation. 2014;37(5):1806-1813. doi:10.1007/s10753-0149911-4

73. Thurner L, Preuss KD, Fadle N, et al. Progranulin antibodies in autoimmune diseases. J Autoimmun. 2013;42:29-38. doi:10.1016/ j.jaut.2012.10.003

74. Mills JA. Systemic lupus erythematosus. $N$ Engl J Med. 1994;330 (26):1871-1879. doi:10.1056/NEJM199406303302608

75. Choi MY, Flood K, Bernatsky S, Ramsey-Goldman R, Clarke AE. A review on SLE and malignancy. Best Pract Res Clin Rheumatol. 2017;31(3):373-396. doi:10.1016/j.berh.2017.09.013 
76. Qiu F, Song L, Ding F, et al. Expression level of the growth factor progranulin is related with development of systemic lupus erythematosus. Diagn Pathol. 2013;8:88. doi:10.1186/1746-15968-88

77. Yang $\mathrm{T}$, Zhang $\mathrm{X}$, Chen A, et al. Progranulin Promotes Bleomycin-Induced Skin Sclerosis by Enhancing Transforming Growth Factor-beta/Smad3 Signaling through Up-Regulation of Transforming Growth Factor-beta Type I Receptor. Am J Pathol. 2019;189(8):1582-1593. doi:10.1016/j.ajpath.2019.04.012

78. Abraham DJ, Varga J. Scleroderma: from cell and molecular mechanisms to disease models. Trends Immunol. 2005;26 (11):587-595. doi:10.1016/j.it.2005.09.004

79. Bhattacharyya S, Wei J, Varga J. Understanding fibrosis in systemic sclerosis: shifting paradigms, emerging opportunities. Nat Rev Rheumatol. 2011;8(1):42-54. doi:10.1038/nrrheum.2011.149

80. van den Hoogen F, Khanna D, Fransen J, et al. 2013 classification criteria for systemic sclerosis: an American college of rheumatology/ European league against rheumatism collaborative initiative. Ann Rheum Dis. 2013;72(11):1747-1755. doi:10.1136/annrheumdis2013-204424

81. Miyagawa T, Ichimura Y, Nakamura K, et al. Progranulin overproduction due to constitutively activated c-Abl/PKC-delta/Fli1 pathway contributes to the resistance of dermal fibroblasts to the anti-fibrotic effect of tumor necrosis factor-alpha in localized scleroderma. J Dermatol Sci. 2018;92(2):207-214. doi:10.1016/ j.jdermsci.2018.09.005

82. Hasegawa M, Sato S, Nagaoka T, Fujimoto M, Takehara K. Serum levels of tumor necrosis factor and interleukin-13 are elevated in patients with localized scleroderma. Dermatology. 2003;207(2):141-147. doi:10.1159/000071783

83. Ramirez J, Hernandez MV, Galve J, Canete JD, Sanmarti R. Morphea associated with the use of Adalimumab: a case report and review of the literature. Mod Rheumatol. 2012;22 (4):602-604. doi:10.3109/s10165-011-0550-4

84. Mattozzi C, Richetta AG, Cantisani C, et al. Morphea, an unusual side effect of anti-TNF-alpha treatment. Eur J Dermatol. 2010;20 (3):400-401. doi:10.1684/ejd.2010.0946

85. Kaser A, Zeissig S, Blumberg RS. Inflammatory bowel disease. Annu Rev Immunol. 2010;28:573-621. doi:10.1146/annurevimmunol-030409-101225

86. Atreya I, Atreya R, Neurath MF. NF-kappaB in inflammatory bowel disease. J Intern Med. 2008;263(6):591-596. doi:10.1111/ j.1365-2796.2008.01953.x

87. Thurner L, Stoger E, Fadle N, et al. Proinflammatory progranulin antibodies in inflammatory bowel diseases. Dig Dis Sci. 2014;59 (8):1733-1742. doi:10.1007/s10620-014-3089-3

88. Tian R, Li Y, Yao X. PGRN Suppresses Inflammation and Promotes Autophagy in Keratinocytes Through the Wnt/ beta-Catenin Signaling Pathway. Inflammation. 2016;39 (4):1387-1394. doi:10.1007/s10753-016-0370-y

89. Greb JE, Goldminz AM, Elder JT, et al. Psoriasis. Nat Rev Dis Primers. 2016;2:16082. doi:10.1038/nrdp.2016.82

90. Huang K, Chen A, Zhang X, et al. Progranulin is preferentially expressed in patients with psoriasis vulgaris and protects mice from psoriasis-like skin inflammation. Immunology. 2015;145 (2):279-287. doi:10.1111/imm.12446
91. Zheng P, Li Z, Zhou Z. Gut microbiome in type 1 diabetes: a comprehensive review. Diabetes Metab Res Rev. 2018;34(7): e3043. doi:10.1002/dmrr.3043

92. Oh J, Vidal-Jordana A, Montalban X. Multiple sclerosis: clinical aspects. Curr Opin Neurol. 2018;31(6):752-759. doi:10.1097/ WCO.0000000000000622

93. Chalah MA, Ayache SS. Is there a link between inflammation and fatigue in multiple sclerosis? J Inflamm Res. 2018;11:253-264. doi:10.2147/JIR.S167199

94. Pawlitzki M, Sweeney-Reed CM, Bittner D, et al. CSFProgranulin and Neurofilament Light Chain Levels in Patients With Radiologically Isolated Syndrome-Sign of Inflammation. Front Neurol. 2018;9:1075. doi:10.3389/fneur.2018.01075

95. Jiao J, Herl LD, Farese RV, Gao FB. MicroRNA-29b regulates the expression level of human progranulin, a secreted glycoprotein implicated in frontotemporal dementia. PLoS One. 2010;5(5): e10551. doi:10.1371/journal.pone.0010551

96. Chen L, Li Q, Wang J, et al. MiR-29b-3p promotes chondrocyte apoptosis and facilitates the occurrence and development of osteoarthritis by targeting PGRN. J Cell Mol Med. 2017;21 (12):3347-3359. doi:10.1111/jcmm.13237

97. Xie W, Lu Q, Wang K, et al. miR-34b-5p inhibition attenuates lung inflammation and apoptosis in an LPS-induced acute lung injury mouse model by targeting progranulin. J Cell Physiol. 2018;233(9):6615-6631. doi:10.1002/jcp.26274

98. Miyakawa S, Sakuma H, Warude D, et al. Anti-sortilin1 Antibody Up-Regulates Progranulin via Sortilin1 Down-Regulation. Front Neurosci. 2020;14:586107. doi:10.3389/fnins.2020.586107

99. Xia Q, Zhu S, Wu Y, et al. Intra-articular transplantation of atsttrin-transduced mesenchymal stem cells ameliorate osteoarthritis development. Stem Cells Transl Med. 2015;4(5):523-531. doi:10.5966/sctm.2014-0200

100. Bateman A, Bennett HP. The granulin gene family: from cancer to dementia. Bioessays. 2009;31(11):1245-1254. doi:10.1002/ bies. 200900086

101. Matsumura N, Mandai M, Miyanishi M, et al. Oncogenic property of acrogranin in human uterine leiomyosarcoma: direct evidence of genetic contribution in in vivo tumorigenesis. Clin Cancer Res. 2006;12(5):1402-1411. doi:10.1158/1078-0432.CCR-05-2003

102. Altmann C, Vasic V, Hardt S, et al. Progranulin promotes peripheral nerve regeneration and reinnervation: role of notch signaling. $\mathrm{Mol}$ Neurodegener. 2016;11(1):69. doi:10.1186/s13024-016-0132-1

103. Neill T, Buraschi S, Goyal A, et al. EphA2 is a functional receptor for the growth factor progranulin. $J$ Cell Biol. 2016;215 (5):687-703. doi:10.1083/jcb.201603079

104. Kuksin CA, Minter LM. The Link between Autoimmunity and Lymphoma: does NOTCH Signaling Play a Contributing Role? Front Oncol. 2015;5:51. doi:10.3389/fonc.2015.00051

105. Khanmohammadi S, Shabani M, Tabary M, Rayzan E, Rezaei N. Lymphoma in the setting of autoimmune diseases: a review of association and mechanisms. Crit Rev Oncol Hematol. 2020;150:102945. doi:10.1016/j.critrevonc.2020.102945

106. Huang J, Liang Y, Tian W, et al. Antitumor Activity and Mechanism of Robustic Acid from Dalbergia benthami Prain via Computational Target Fishing. Molecules. 2020;25(17):254. doi:10.3390/molecules 25173919 


\section{Publish your work in this journal}

The Journal of Inflammation Research is an international, peerreviewed open-access journal that welcomes laboratory and clinical findings on the molecular basis, cell biology and pharmacology of inflammation including original research, reviews, symposium reports, hypothesis formation and commentaries on: acute/chronic inflammation; mediators of inflammation; cellular processes; molecular mechanisms; pharmacology and novel anti-inflammatory drugs; clinical conditions involving inflammation. The manuscript management system is completely online and includes a very quick and fair peerreview system. Visit http://www.dovepress.com/testimonials.php to read real quotes from published authors. 